\title{
Metabolic changes from liver regeneration after metastatic colorectal cancer surgery
}

\author{
Anton Burlaka ${ }^{1}$, Vitaliy Zvirych ${ }^{1}$, Volodymyr Dorozhinskiy ${ }^{1}$, Oleg Vasiliev ${ }^{1}$, \\ Anastasiya Vovk ${ }^{2}$, Valeriy Orel ${ }^{1}$, Olena Kolesnik ${ }^{1}$
}

Background. The pathological effect of the surgical ischemia-reperfusion technique is a cause for the development of liver damage and occurrence of acute liver failure during the postoperative period in cancer patients after major resection as well as after transplantation. Despite the urgency of the problem, most of the pathological processes that accompany the above-mentioned conditions are still only incompletely studied.

Objective. To investigate the mechanisms of the damaging effects of stress reaction after liver resection, to evaluate the functional state of residual liver tissue and to find ways to influence the regeneration and apoptosis of the resected liver, which in the future would make the liver surgery safer.

Methods. The results of treatment of 15 patients with colorectal cancer ( $\mathrm{mCRC}$ ) and liver metastases, who had undergone surgery in the period from December 2015 to November 2016 at the National Cancer Institute. Determination of the state of the detoxifying function of hepatocytes and the coupling of biological oxidation and phosphorylation in the mitochondria of hepatocytes were performed by electron paramagnetic resonance at the temperature of liquid nitrogen.

Results. It has been found that the levels of the oxidized and low-spin forms of cytochrome P-450 in the catalytic cycle of the detoxification system were $0.33 \pm 0.08$ relative units and $1.11 \pm 0.13$ relative units, respectively (the normal levels are $0.59 \pm 0.03$ relative units and $2.56 \pm 0.02$ relative units). Also, an increase registered in the levels of formation of complexes of NO-FeS-proteins up to the values of $0.33 \pm 0.08$ relative units (the normal level is $0.14 \pm$ 0.07). We believe that such changes are the cause of disturbances in the energy function of mitochondria and the formation of cell hypoxia, which correlates with an increase of lactate levels in tissue of the future liver remnant. The liver remnant levels are $>2.00 \mathrm{mM} / \mathrm{g}$.tissue, while the normal is $1.80 \pm 0.26 \mathrm{~mm} / \mathrm{g} \cdot$ tissue. This confirms the functioning of hepatocytes under hypoxic conditions.

Conclusions. In the residual liver tissue, we have found a decrease in detoxification system deficiency and the energy supply of hepatocytes. In hepatocytes of the future liver remnant, reprogramming of the metabolism of mitochondria from oxidative phosphorylation in glycolysis is registered, resulting in the formation of cell hypoxia and an increase in levels of lactate and superoxide radicals. To assess the degree of ALF in the postoperative period, we suggest use of the levels of lactate, the activity of cytochrome P-450, the levels of formation of complexes of NO and FeS-proteins in mitochondria ETC and the generation rate of SR as the markers.

NOWOTWORY J Oncol 2017; 67, 2: 121-126

Key words: colorectal cancer, liver resection, regeneration, ischemia-reperfusion 


\section{Introduction}

It is known that the liver has a mechanism of specific restoration of its previous volume and functioning in a short period of time after resection or damage [1]. Regeneration of the organ occurs after liver resection or transplantation, as well as after toxic damage. The process of liver regeneration includes hyperplasia of all types of liver cells. It is known that hepatocyte replication starts at the moment of major liver resection, but the replication of non-parenchymatous cells (endothelial, Kupffer cells and bile duct cells) activates later [2].

Intensive studies on liver regeneration have been carried out over the last two decades, but the mechanisms responsible for liver volume regeneration remain unknown. It is clear that the liver maintains a precise balance between the absence and excess of its parenchyma by regulating the processes of growth [3]. The recent results of clinical and experimental studies of the pathophysiology of resected liver only insufficiently reveal new aspects of the processes of liver failure and regeneration [4].

The pathological effect of the surgical ischemia-reperfusion technique is a cause of the development of liver damage and the occurrence of acute liver failure in the postoperative period in cancer patients after major resection, as well as after transplantation. Despite the urgency of the problem, most of the pathological processes that accompany the above-mentioned conditions are still insufficiently studied.

The aim of this work was to investigate the mechanisms of the damaging effects of stress reaction after liver resection, to evaluate the functional state of residual liver tissue and to find ways to influence regeneration and apoptosis of resected liver cells, which in the future would make liver surgery safer. Understanding liver regeneration mechanisms will allow correction and prevention of the acute conditions of toxic or infectious injury of the organ.

\section{Materials and methods}

The results of treatment of 15 patients with colorectal cancer ( $\mathrm{mCRC}$ ) and liver metastases, who had undergone surgery in the period from December 2015 to November 2016 at the National Cancer Institute (Tab. I) were studied. Patients received chemotherapy in accordance with approved international and national standards and clinical research protocols.

The protocol of surgical intervention included intraoperative ultrasound examination of liver and compliance with the principles of anatomic resections. Liver surgery was performed using the "Extra-Glissonian approach". When anatomical segmentectomies were necessary, "Selective pedicle clamping" was used and during the anatomical sectionectomy, the "Hanging Maneuver". Transection of liver
Table I. Characteristics of patients

\begin{tabular}{|c|c|}
\hline Indicators & Number (n) \\
\hline Primary tumor site (rectal/colon) & $3 / 12$ \\
\hline Simultaneous/staged resection & $2 / 13$ \\
\hline Synchronous/metachronous liver metastases & $4 / 11$ \\
\hline Metastases in other sites at the time of liver resection: & 4 \\
\hline Lungs & 3 \\
\hline Abdomen & 1 \\
\hline $\begin{array}{l}\text { Neoadjuvant chemotherapy before the liver surgery } \\
\text { (number of courses): }\end{array}$ & 14 \\
\hline FOLFOX-6 $(\leq 3)$ & 4 \\
\hline FOLFOX-6 $(>3)$ & 3 \\
\hline XELOX $(\leq 3)$ & 2 \\
\hline XELOX (> 3) & 3 \\
\hline FOLFIRI $(\leq 3)$ & 2 \\
\hline \multicolumn{2}{|l|}{ Patient condition on the ASA scale: } \\
\hline$|-| \mid$ & 11 \\
\hline III & 3 \\
\hline \multicolumn{2}{|l|}{ Number of resected liver segments: } \\
\hline$\leq 3$ & 2 \\
\hline$>3$ & 12 \\
\hline Duration of ischemia-reperfusion (avg. value) (min.) & $31.2 \pm 9.6$ \\
\hline Duration of surgery (avg. value) (min.) & $154.8 \pm 19.2$ \\
\hline
\end{tabular}

parenchyma was performed using an ultrasound dissector, mono- and bipolar coagulators.

Samples of parenchyma of the future liver remnant were taken during and after surgical intervention within 1 hour after completion of the resection. Lactate in the liver tissue was determined spectrophotometrically [5]. Determination of the state of the detoxifying function of hepatocytes and the coupling of biological oxidation and phosphorylation in the mitochondria of hepatocytes was performed using electron paramagnetic resonance (EPR) at the temperature of liquid nitrogen. For that purpose, $500 \mathrm{mg}$ of liver tissue were injected into a special press-mold and frozen in liquid nitrogen. The samples were studied using a computerized spectrometer EPR PE-1307. Qualitative and quantitative changes in the functioning of the mitochondrial electron transport chain were determined by EPR signal location (g-factor of spectroscopic splitting); also, an amplitude of the signal in the control and experimental samples of tissues was measured. We investigated the state of FeS-proteins in the first electron transport complex of ETC mitochondria (g-factor 1.94), the level of flavin semiquinone in ETC mitochondria (g-factor 2.00), the dynamic of formation of NO complexes with FeS-proteins in ETC mitochondria of $\mathrm{N}$-type (g-factor 2.03), the dynamic of formation of structural 
changes in ETC mitochondria by forming a triplet signal (g-factor 2.007). The state of the detoxification system in hepatocytes was evaluated by the activity of cytochrome P-450 (g-factor 2.25 and 2.42).

Statistical analysis of the data was performed using the package STATISTICA 6.0 (StatSoft, USA, 2001). Normality of the distribution of variables was checked by a Shapiro-Wilk test. When checking the statistical hypotheses, $p=0.05$ was chosen as the critical significance level. Given that the most of the parameters had a distribution different from the normal, to describe the selective distribution, the median (Me) and lower (25\%) and upper (75\%) quartiles were specified: Me (25\%; 75\%). When checking the statistical hypotheses, $p=0.05$ was chosen as the critical significance level.

\section{Results and discussion}

Hepatocytes, are very sensitive to ischemic conditions and it is known that ischemia-reperfusion is one of the main factors in acute liver failure after major liver resections, especially at normometric ischemia. Most of the pathological changes that occur in hepatocytes at anoxic conditions are detected in mitochondria. Disturbance of $\mathrm{O}_{2}$ metabolism in the mitochondria respiratory chain leads to changes in electron transport and mitochondrial respiration. Under such conditions, a sharp decrease in the production of pyrimidine nucleotides occurs that in its turn leads to higher accumulation of levels of intracellular NADH $\backslash \mathrm{NAD}^{+}$. Changes in the processes of oxidative phosphorylation lead to activation of depletion of intracellular ATP, acceleration of glycolysis, higher levels of lactate and an increase in the influence of $\mathrm{H}^{+}$, $\mathrm{Na}^{+}$and $\mathrm{Ca}^{2+}$ on homeostasis. The described pathological changes lead to serious disorders of the function of hepatocytes and the liver in general in the postoperative period.

In its turn, reperfusion causes damage to cells by products of incomplete reduction of oxygen - radical oxygen species (ROS), in particular, superoxide radicals at re-admission of $\mathrm{O}_{2}$ in ischemized tissues. ROS are produced both within cells (by mitochondria) and by extracellular sources (NOX, iNOS of neutrophils, platelets, lymphocytes) [6].

According to the results of our own researches, in tissues of the future liver remnant, the functional exhaustion of the detoxification ability of hepatocytes has been revealed. It has been found that levels of the oxidized and low-spin forms of cytochrome P-450 in the catalytic cycle of the detoxification system were $0.33 \pm 0.08$ relative units and $1.11 \pm 0.13$ relative units, respectively (the normal levels of $0.59 \pm 0.03$ relative units and $2.56 \pm 0.02$ relative units) (Fig. 1 ).

The mitochondria of these cells are functioning when the coupling of oxidation and phosphorylation in NAD.H-ubiquinone oxidoreductase is disturbed. The level of FeS-protein $\mathrm{N}-2$ in this electron transport complex was $0.32 \pm 0.06$ relative units while the normal one was $0.61 \pm 0.09$ relative units (Fig. 2 ).

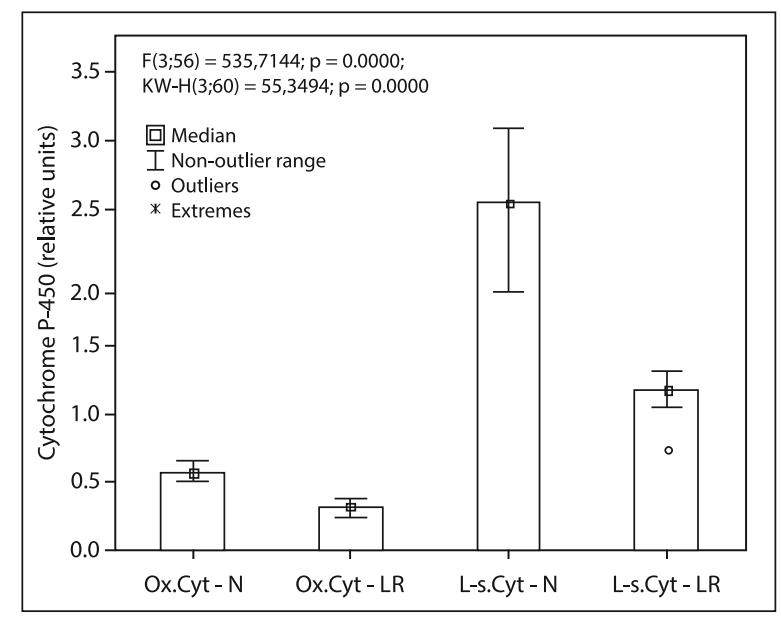

Figure 1. State of the detoxification system of hepatocytes in patients with $\mathrm{mCRC}$ after liver resection. Ox. CYP450 - oxidized cytochrome; L. Sp. CYP450 - low-spin cytochrome; N - indicators of liver tissue in normal state; LR - parameters of liver tissue after resection

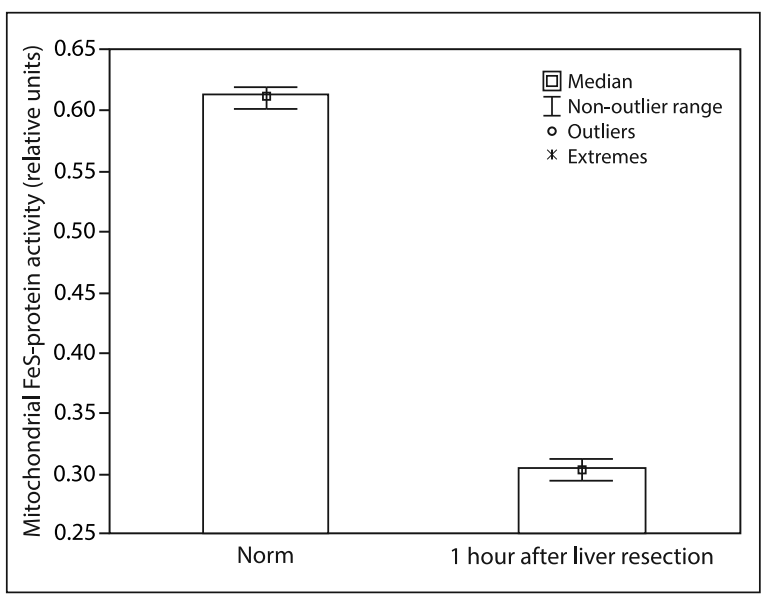

Figure 2. State of the electron transport chain of the mitochondria of liver hepatocytes in patients with mCRC ( 1 hour after major liver resection and in the normal state)

Also, an increase in the levels of formation of complexes of NO-FeS-proteins up to the values of $0.33 \pm 0.08$ relative units registered (the normal level of $0.14 \pm 0.07$ ). We believe that such changes are the cause of the disturbance of energy production in mitochondria and the formation of cell hypoxia. This correlates with an increase in levels of lactate in tissue of future liver remnant to levels $>2.00 \mathrm{mM} / \mathrm{g}$.tissue, while the normal one is $1.80 \pm 0.26 \mathrm{~mm} / \mathrm{g}$.tissue, and confirms the functioning of hepatocytes under hypoxic conditions (Fig. 3).

Mitochondria play a crucial role in the regulation of the death of cells through apoptosis, especially in hepatocytes. Activation of the death receptor induces apoptosis involving caspase-8 through destruction of mitochondrial membrane FeS-proteins and inactivation of the flow of electrons in the 


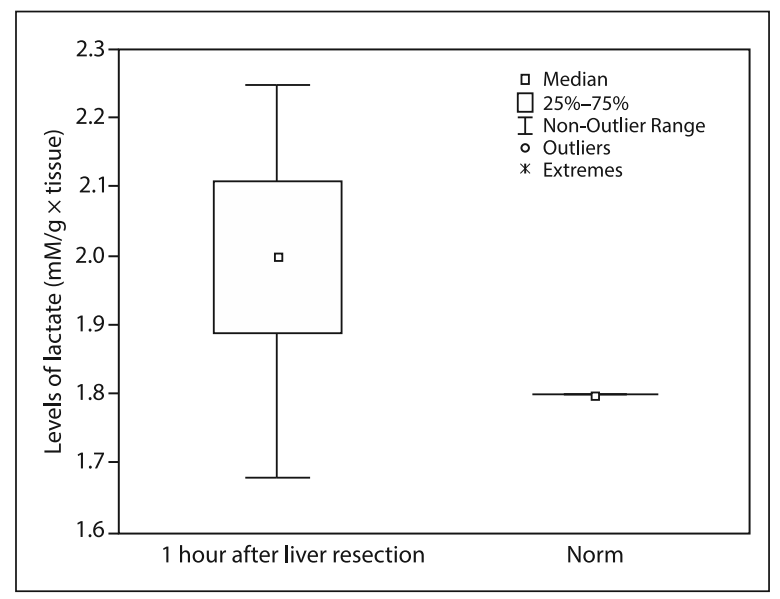

Figure 3. Levels of lactate in liver tissue after major resection in patients with $\mathrm{mCRC}$ (in the normal state and 1 hour after resection)

electron transport chain (ETC), which causes the unregulated generation of superoxide radicals in mitochondria and, finally, the formation of apoptosome. These data indicate that defects in the respiratory chain and an excess of free fatty acids are the key factors that occur during ischemia/ /reperfusion at liver resections. Mitochondrial dysfunction is enhanced by the increase in the levels of generating $\mathrm{SR}$, which initiate formation of a self-reinforcing cycle of formation of radicals, which are formed as a result of lipid peroxidation and are able to inhibit the complexes I and IV of ETC. On the other hand, SR damage mtDNA and FeS-proteins of the respiratory chain that leads to chronic damage of the organelle. Cytochrome P-450 (isoforms CYP2E1 and CYP4A10/4A14) activates the elevated levels of free fatty acids in hepatocytes of resected liver, which leads to enhancement of generation of superoxide radicals and an increase in mitochondrial dysfunction. Mitochondria control the energy production, metabolism, activation of programmed cell death, as well as several specific cell functions, including cell signaling and synthesis of some important biomolecules. Normalization of mitochondrial function is crucial to the functioning and survival of cells, and changes in it, i.e., mitochondrial dysfunction, lead to pathological conditions. Mitochondrial dysfunction causes multi-organ failure after intensive therapy, which often leads to the death of patients. However, there are two main reasons why this fact has not received adequate appraisal by clinicians. First, the main results on the occurrence of mitochondrial dysfunction in cells of organs, susceptible to damage of mitochondria were obtained in animal models, using intensive therapy (liver, kidneys, heart, lungs, intestines, brain). Second, there is still no clear therapeutic strategy on how to restore mitochondrial function.

\section{Signaling pathways of regeneration and their impact on the growth and metastasis}

Under normal conditions, hepatocytes do not divide and remain in the $\mathrm{G} 0$ phase. After resection or toxic damage of the liver, they enter into the $G 1$ phase. Such changes are triggered by binding receptors of liver cells to tumor necrosis factor $\alpha$ (TNF- $\alpha$ ) and interleukin- 6 (IL-6), which are released by Kupffer cells [7]. Several molecular structures are responsible for activation of hepatocyte growth factor (HGF), thereby initiating transition of cells from the G1 phase to the $S$, including the epidermal growth factor (EGF) and transforming growth factor $\alpha$ (TGF- $\alpha$ ) [8]. These factors stimulate DNA replication and mitosis, interacting with the corresponding receptors [9]. It is proven that TGF- $\beta 1$ causes inhibition of hepatocyte proliferation, while in a normal liver the growth factor TGF- $\beta$ has the agonistic effects. At the initial stages of regeneration the signals of HGF are more intensive, as compared to TGF- $\beta$, and at the final stages of regeneration the restoration of the initial balance occurs $[9,10]$. The factor of liver resection is a powerful stimulus to its regeneration. After surgical intervention, an increase in the rate and pressure of portal blood flow on hepatocytes and sinusoidal endothelial cells occurs that initiates the regeneration and regulates the size of the liver. Resection stress is considered to be a powerful impulse to regeneration, regulation of volume and growth, as well as atrophy [11]. In their turn, the hemodynamic factors potentiate the action of resection stress in liver, stimulating generation of nitrogen oxide (NO), which in turn activates a cascade of liver regeneration.

The leading role in the regulation of metabolism in the intercellular matrix of liver parenchyma belongs to superoxide radicals (SR). The latter are redox-dependent molecules actively regulating the occurrence and development of pathological processes. These properties of SR may be changed in the dynamics of pathological processes, as well as the structure of intersystem interactions. SR are able to show not only cytotoxic properties, but they can also act as secondary messengers, taking part in support of physicochemical properties of biological membranes, regulation of intracellular redox systems, activity of protein kinases and cell proliferation, differentiation, apoptosis. In recent years, experts in advanced international experimental and clinical cancer centers publish evidence of the importance of SR and their key role in the regulation of intercellular matrix and matrix metalloproteinases. Processes of overregulation of biological structures by SR are cyclic in nature, because microcirculatory disorders and trans-capillary exchange cause tissue hypoxia and activate a number of angiogenic factors, metalloproteinase (MMP-2 and MMP-9), TNF- $\alpha$, TNF- $\beta$, that affect the balance of anti- and pro-inflammatory cytokines. 
Initiation and progression of tumor growth in the liver is a multifactorial molecular process that involves complex signaling pathways (RAS/RAF-MAPK, phosphatidylinositol-3 kinase (PI3K)/AKT, WNT/ $\beta$-catenin, insulin-like growth factor (IGF), hepatocyte growth factor (HGF)/c-MET and the growth factors activating angiogenesis pathways) [12]. It has been found that cellular and molecular changes in the postoperative period of the patients who have undergone liver resection (response to surgical stress, damage due to ischemia-reperfusion) can initiate tumor growth kinetics and thus cause rapid progression [13].The molecular factors, which are produced in the liver tissue because of surgical stress, can be a powerful stimulus for tumor growth. Regeneration of the liver after resection is activated by the microenvironment and a variety of growth factors, cytokines, responsible for the activation and growth of malignant cells (proliferation, migration and neoangiogenesis) [14]. During hepatocyte proliferation, the endocrine, autocrine and paracrine processes are working, which also can stimulate dormant micrometastasis and tumor growth [15]. One of the main results of surgical intervention in the zone of the future liver remnant is the fast release of SR, NO, TNF- $\alpha$, $\mathrm{TGF}-\beta, \mathrm{HGF}, \mathrm{EGF}$, interleukin 6 , angiogenic factors, modifying the microenvironment of dormant tumor cells and stimulating the growth and metastasis of tumor [16]. Tumor growth is a kind of balance between the concentration of growth factors and cytokines in the microenvironment, and stimulation of neoangiogenesis [17]. Inhibitors of angiogenesis, proteins of extracellular matrix and their fragments act as stabilizers of the quiescent state of tumor cells [18].

\section{Clinical manifestations of the regenerative ability of liver and possibilities for correction}

After liver resection, the residual parenchyma of the organ reacts by activation of hyperplasia. After major liver resection, the "survived" hepatocytes quickly restart the cell cycle. Thus, the liver restores its volume through proliferation. There are two generally recognized hypothetical ways to activate liver regeneration [19]. One of them is the generation of stress signals by increasing the demand for energy per liver after resection of the organ [20]. The other is to activate liver regeneration by changing hemodynamic parameters. The correlative connection between the blood flow and liver regeneration has been proven, but the mechanisms of this interaction are still to be explained. Irregular proliferation of dormant tumor cells after liver resection requires activation of many intracellular pathways of overregulation, including cascades involved in "survival", proliferation and cell cycle. Induction of redox pathways in the cell proliferation process requires significant energy.

Most of studies of liver tissue regeneration focused on the treatment of human liver diseases, resulting from surgery, chronic liver diseases and cancer problems. In the past few decades, liver resections and transplantation have allowed many problems to be solved, however acute liver failure (ALF) in the postoperative period remains a major cause of death of patients, which occurs in 10\% [1]. Most often for evaluation of ALF in clinical practice the 50-50 criteria is used, including the assessment of INR $<50 \%$ and total level of serum bilirubin $>50 \mu \mathrm{mol} / \mathrm{L}(>2.9 \mathrm{mg} / \mathrm{dL}$ ) on postoperative day 5 [1]. In 2011, the International Study Group of Liver Surgery (ISGLS) described and proposed the determination and scale of ALF [1].This type of assessment remains of current interest only because of a considerable increase in the understanding of the clinical aspects of liver regeneration in patients with chronic diseases of the organ. However, it cannot fully reveal the potential risks of ALF in postoperative period related with the functional disorders of hepatocytes.

Progression of the disease and its metastasis after liver resection so far remains an important problem. An adequate surgical intervention based on the principles of saving organ and in accordance with modern methods of radiological investigation is considered to be the best strategy. In the last decade, the greatest contribution in this area has been made in the development of radiological technologies of imaging the tumor and its metastases. The modern possibilities of $C T$ and MRI with the use of hepato-specific contrast, software and diffuse-weighted analysis are able to improve significantly the accuracy of detection of small malignancies [21]. There are certain problems with cirrhotic altered liver when malignant neoplasms $\leq 1 \mathrm{~cm}$ are difficult to distinguish from the regeneration sites. Ultrasound diagnostics (Ultrasound) with contrast allows differential diagnosis in this area, adding to the data of MRI and CT [22]. In difficult cases, it is important to combine several methods. In addition, the use of intraoperative Ultrasound can increase the percentage of radically operated patients (R0).

It is known that recurrence of malignant neoplasms in the liver is a poor prognostic factor, and surgical and therapeutic methods of treatment depend on the anatomical area, functional status and general status of the patient. As modalities for the treatment of recurrences of malignant neoplasms in the liver and its metastatic lesions, repeated resections of the organ, transplantation, local ablation and transcatheter arterial chemoembolization (TACE) were used [23]. Repeated liver resections are considered to be the best method in this case; according to different authors, the resectability ranges vary from 10 to $77 \%$ [24]. A longer interval between the surgical treatment and recurrence of the disease is associated with improved survival [25]. TACE is regarded as the best method for unresectable forms of recurrent hepatocellular carcinoma [26]. Generally, multimodal therapy for recurrent malignant liver tumors (TACE, local ablation and re-resection) are able to improve the 5 -year survival rate to $20 \%$ [27]. The approaches to destruc- 
tion of malignant tumors without affecting the regenerative ability of liver regeneration is suggested. These include targeted therapy and immunotherapy. It is known that liver regeneration involves a complex of molecular processes, including angiogenesis. Synthesis of angiogenic products in the future liver remnant reaches maximal concentrations 48-72 hours after the moment of resection, which in turn can lead to an increase in dilatation and permeability of blood vessels. However, inhibition of angiogenesis with the use of angiostatin affects both the process of growth of blood vessels and the regeneration of the liver [28].

\section{Conclusions}

A decrease in the efficiency of the detoxification system and energy supply of hepatocytes is found in liver remnant. We found reprogramming of mitochondrial metabolism after the liver resection with oxidative phosphorylation in glycolysis, which resulted in the formation of cell hypoxia and an increase in the levels of lactate and superoxide radicals. To assess the degree of ALF in the postoperative period, we suggest use of the levels of lactate, activity of cytochrome P-450, levels of formation of complexes of NO and FeS-proteins in ETC mitochondria and the generation rate of SR as the markers. Using innovative technologies can change the therapeutic strategy for liver dysfunction, such as ALF and the'small-for-size' liver syndrome. Particular attention must be addressed to mechanisms that cause cell death and dysfunction of organs and prospective therapeutic strategies aimed to restore the mitochondrial functions.

\section{Conflict of interest: none declared}

\section{Anton Anatoliyovych Burlaka, MD, PhD}

National Cancer Institute

Lomonosova 33/43, 03022 Kyiv, Ukraine

e-mail:nir.burlaka@gmail.com

Received: 8 Mar 2016

Accepted: 13 Apr 2016

\section{References}

1. Michalopoulos GK. Liver regeneration. J Cell Physio/ 2007; 213: 286-300.

2. Clavien PA, Petrowsky H, DeOliveira ML et al. Strategies for safer liver surgery and partial liver transplantation. N Engl J Med 2007; 356: 1545-1559.

3. Kholodenko IV, Yarygin KN. Cellular mechanisms of liver regeneration and cell-based therapies of liver diseases. Biomed Res Int 2017; 2017: 8910821.
4. Duncan AW, Soto-Gutierrez A. Liver repopulation and regeneration: new approaches to old questions. Curr Opin Organ Transplant 2013; 18: 197-202.

5. McGehee JC, Tanner CJ, Houmard JA. A comparison of methods for estimating the lactate threshold. JStrength Cond Res 2005; 19: 553-558.

6. He N, Jia JJ, Li JH et al. Remote ischemic perconditioning prevents liver transplantation-induced ischemia/reperfusion injury in rats: Role of ROS/RNS and eNOS. World J Gastroenterol 2017; 23: 830-841.

7. Kwon YJ, Lee KG, Choi D. Clinical implications of advances in liver regeneration. Clin Mol Hepatol 2015; 21: 7-13.

8. Huang L, Fu L. Mechanisms of resistance to EGFR tyrosine kinase inhibitors. Acta Pharm Sin B 2015; 5: 390-401.

9. Zheng ZY, Weng SY, Yu Y. Signal molecule-mediated hepatic cell communication during liver regeneration. World J Gastroenterol 2009; 15: 5776-5783.

10. Michalopoulos GK. Liver regeneration after partial hepatectomy: critical analysis of mechanistic dilemmas. Am J Pathol 2010; 176: 2-13.

11. Cai YL, Song PP, Tang W et al. An updated systematic review of the evolution of ALPPS and evaluation of its advantages and disadvantages in accordance with current evidence. Medicine 2016; 95: e3941.

12. Paschos KA, Bird NC. Liver regeneration and its impact on post-hepatectomy metastatic tumour recurrence. Anticancer Res 2010; 30: 2161-2170.

13. Man K, Lo CM, Xiao JW et al. The significance of acute phase small-for-size graft injury on tumor growth and invasiveness after liver transplantation. Ann Surg 2008; 247: 1049-1057.

14. Shi JH, Line PD. Effect of liver regeneration on malignant hepatic tumors. World J Gastroenterol 2014; 20: 16167-16177.

15. Marín-Hargreaves G, Azoulay D, Bismuth H. Hepatocellular carcinoma: surgical indications and results. Crit Rev Oncol Hematol 2003; 47: 13-27.

16. He G, Karin M. NF- $\kappa$ B and STAT3 - key players in liver inflammation and cancer. Cell Res 2011; 21: 159-168.

17. Gaudio E, Carpino G, Cardinale V et al. New insights into liver stem cells. Dig Liver Dis 2009; 41: 455-462.

18. O'Reilly MS, Boehm T, Shing Y et al. Endostatin: an endogenous inhibitor of angiogenesis and tumor growth. Cell 1997; 88: 277-285.

19. Abshagen K, Eipel C, Vollmar B. A critical appraisal of the hemodynamic signal driving liver regeneration. Langenbecks Arch Surg 2012; 397: 579-590.

20. Crumm S, Cofan M, Juskeviciute $E$ et al. Adenine nucleotide changes in the remnant liver: An early signal for regeneration after partial hepatectomy. Hepatology 2008; 48: 898-908.

21. Murakami T, Okada M, HyodoT.CT versus MR imaging of hepatocellular carcinoma: toward improved treatment decisions. Magn Reson Med Sci 2012; 11: 75-81.

22. Guang $Y, X i e ~ L$, Ding $H$ et al. Diagnosis value of focal liver lesions with SonoVue ${ }^{\oplus}$-enhanced ultrasound compared with contrast-enhanced computed tomography and contrast-enhanced MRI: a meta-analysis. J Cancer Res Clin Oncol 2011: 137: 1595-1605.

23. Rampone B, Schiavone B, Martino A et al. Current management strategy of hepatocellular carcinoma. World J Gastroenterol 2009; 15: 3210$-3216$.

24. Neeleman N, Andersson R. Repeated liver resection for recurrent liver cancer. Br J Surg 1996; 83: 893-901.

25. Hargreaves GM, Adam R, Bismuth $H$. Results after nonsurgical local treatment of primary liver malignancies. Langenbecks Arch Surg 2000; 385: 185-193.

26. Marín-Hargreaves G, Azoulay D, Bismuth H. Hepatocellular carcinoma: surgical indications and results. Crit Rev Oncol Hematol 2003; 47: 13-27.

27. Minagawa M, Makuuchi M, Takayama T et al. Selection criteria for repeat hepatectomy in patients with recurrent hepatocellular carcinoma. Ann Surg 2003; 238: 703-710.

28. Pagano D, Spada M, Parikh V et al. Liver regeneration after liver resection: clinical aspects and correlation with infective complications. World J Gastroenterol 2014; 20: 6953-6960. 